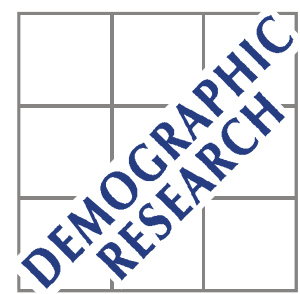

Demographic Research a free, expedited, online journal of peer-reviewed research and commentary in the population sciences published by the Max Planck Institute for Demographic Research Doberaner Strasse 114 · D-18057 Rostock · GERMANY www.demographic-research.org

DEMOGRAPHIC RESEARCH

VOLUME 3, ARTICLE 10

PUBLISHED 7 NOVEMBER 2000

www.demographic-research.org/Volumes/Vol3/10/

DOI: 10.4054/DemRes.2000.3.10

\title{
Measuring Local Heterogeneity with 1990 U.S. Census Data
}

\author{
Kenneth W. Wachter
}

\section{David A. Freedman}

(C) 2000 Max-Planck-Gesellschaft. 


\title{
Measuring Local Heterogeneity with 1990 U.S. Census Data
}

\author{
Kenneth W. Wachter ${ }^{1}$ \\ David A. Freedman ${ }^{2}$
}

\begin{abstract}
A sample covering 204,394 blocks from the 1990 U.S. Census permits measurement of residual heterogeneity from local area to local area after controlling by stratification for demographic characteristics such as race, ethnicity, age, sex as well as geographic characteristics such as region and place-type. The local areas have populations on the order of 10,000 people. The variables studied are four indices of enumeration difficulty. The results show that variance due to heterogeneity from area to area is comparable to (if not larger than) variance from stratum to stratum and can be expected to dominate sampling variance - especially with samples as large as the ones used in the U.S. Census Bureau's Post-Enumeration Surveys. These findings constrain the viable estimation strategies that could be employed for local tallies in the U.S. 2000 Census.
\end{abstract}

\footnotetext{
${ }^{1}$ Professor of Demography and of Statistics, University of California, Berkeley, CA 94720

${ }^{2}$ Professor of Statistics, University of California, Berkeley, CA 94720
} 


\section{Introduction}

In the United States, census counts are used to apportion congressional seats to states, and to draw the boundaries of electoral districts within states ("redistricting"). The counts also enter the formulas for allocating tax funds to states, counties, cities, and smaller jurisdictions. Thus, the census has some effect on the distribution of power and money [Skerry 2000]. Controversy over proposed statistical adjustments of population counts from decennial censuses has stimulated an extended program of demographic research over twenty years [see 4]. These issues have been brought again to the fore by the current Director of the U.S. Census Bureau, Kenneth Prewitt.

In considering whether to certify adjusted or unadjusted counts as the official census counts, Prewitt directs attention to the problem of geographical heterogeneity in quality of coverage, which limits the accuracy of small-area estimates; he acknowledges the renewed importance of data from 1990, recognizing that decisions will be made before much of the data from the 2000 Census evaluation process will become available: he favors certifying the adjusted counts, barring some unforeseen developments when the data are collected and analyzed [Prewitt 2000]. Because the U.S. Supreme Court ruled that federal law mandates the use of unadjusted population counts for apportionment, the impact of certification will be on the use of census data for redistricting within states, and the allocation of tax funds to state and substate jurisdictions [Brown et al. 1999].

A large data set for studying geographical heterogeneity in quality of coverage for substate areas as well as for states was assembled around 1990 by the U.S. Census Bureau in its P-12 Evaluation Project. However, most analysis was directed toward state-by-state heterogeneity. In this paper, we analyze the scale of substate heterogeneity as revealed by the P-12 data, to provide scientific background for the political decisions at stake in the Prewitt report.

The issue of heterogeneity should be viewed in the broader statistical context of small-area estimation. Classical statistical sampling theory is about inferences upward from the part to the whole, from sample to population. Accuracy is limited by the size of the sample, essentially through the square root of the sample size. In small-area estimation, the situation is different. The aim is to make inferences sideways from a few parts to all other parts. The plan for the U.S. Census in 2000 calls for extrapolating sideways from a sample of 12,000 block clusters to separate estimates of census undercount for tens of thousands of local areas and each of 5 million inhabited Census blocks. Accuracy is limited not only by sample size but also fundamentally by the amount of heterogeneity from local area to local area. The square root law ceases to apply even if all data-processing can be done without error.

It is standard practice to apply uniform ratio estimators and other small-area techniques only 
after stratifying on available variables like age, sex, and race [Ghosh and Rao 1994]. Through stratification some heterogeneity is removed, leaving residual heterogeneity which at some point still imposes diminishing returns on the gains in accuracy achievable from larger sample size. Before 1990, little was known about levels of residual heterogeneity and the pace of diminishing returns to sample size. Since then, interest in census adjustment has led to a series of studies in the United States [see 4], principally focussed on state-to-state heterogeneity in various indices of enumeration difficulty. The U.S. Census Bureau created, in its P-12 Evaluation Project, a unique data set suitable for studying local as well as state-level heterogeneity. The present study exploits P-12 to derive the first - albeit somewhat tentative - measurements of residual heterogeneity for local areas containing on the order of 10,000 people each.

The measurements of heterogeneity in this study provide a benchmark for assessing smallarea undercount estimation in the census. The issues are summarized in [Prewitt 2000], with extensive references; for another perspective, see [Brown et al. 1999]. An underlying probability model is useful for distinguishing the effects of geographical heterogeneity treated here from other components of error [Freedman, Stark and Wachter 2000]. As well as playing a role in discussions of Census adjustment, the measurements in the present study also bear on the likely accuracy of small-area estimation in many other applications. They follow, in an American context, on the new scientific interest in structural properties of geographical heterogeneity kindled by [Le Bras 1993].

Several questions are frequently asked about research in this area. (i) Why study indices of enumeration difficulty rather than undercounts themselves? (ii) Can residual heterogeneity not be eliminated by finer stratification? (iii) What about other datasets?

(i) The Census does not measure its own undercount. Surveys that do measure undercounts, large as they are, are much too small to measure heterogeneity at any fine geographical scale. Problems with data quality in the 1990 Post-Enumeration Survey (PES) also restrict its usefulness for appraising heterogeneity. Data from the 2000 PES, renamed "Accuracy and Coverage Evaluation" (ACE), will not be available for some time, and research projects to assess the data quality in ACE have uncertain completion dates.

(ii) Possibilities for finer stratification are limited. For Census Bureau purposes, only variables recorded for all respondents on Census short forms are usable for stratification. Moreover, there is little evidence to show that doubling or tripling the number of post-strata would achieve any marked reduction in heterogeneity; we return to this point, below.

(iii) Other publicly available data sets known to us lack one or another key feature of P-12. The U.S. Public Use Microdata Samples (PUMS) only identify geographical location down to "Public Use Microdata Areas" (PUMAs) with more than 100,000 people each. The Census Bureau's 
Summary Tabulation Files have precise geography but little cross-classification by stratifying variables. The other large U.S. surveys, like the Current Population Survey, are much smaller than P-12. Similar limitations of one kind or another apply to data sets collected in other developed countries. The data for French communes achieve geographical resolution an order of magnitude finer than P-12, but lack stratification variables [Le Bras 1993].

Every silver lining has its cloud, and P-12 is no exception. The P-12 data were aggregated by the Bureau in a data-dependent way into "superblocks," in order to protect the confidentiality of the respondents. Superblocks range in size from a city block in Manhattan to some large swath of rural Wyoming. The data we have are based on superblocks: our summary statistics show the heterogeneity in these units, thereby averaging across a full spectrum of more familiar geography. The data suggest, however, that a typical superblock represents a locality whose order of size is 10,000 inhabitants, and our results are best interpreted on that geographic scale. More formal arguments are postponed to the Appendix. 


\section{Heterogeneity}

\subsection{The Measure $\hat{H}$}

This section presents our measure of heterogeneity, and procedures for estimating it from the data. Throughout this discussion we restrict attention to a single variable (e.g., the proportion of persons living in multi-unit housing) and to a single demographic group, for example, Hispanic women aged 20-29. Our object of study is variability from local area to local area within broader areas. We need some terminology and notation to clarify the distinctions.

Territory is our name for one of the broader areas, and each territory spans many local areas. The territories are determined by the design of the underlying estimation project and datacollection effort. In small-area estimation with uniform ratio estimators, all small areas within one territory are assigned a common "uniform" estimated value, based on aggregating across the territory; in census applications, estimates are uniform within combinations of territory and demography called "post-strata." Typically, a territory consists of all places of a particular type across some regional subdivision of the country. In P-12, the central cities in New England are an example of a territory.

Each territory is dissected into localities: examples might be the Back Bay, North End, or Beacon Hill in Boston; or Colorado's Snowmass Mountain Basin, Sangre de Cristos foothills, etc. The estimation project itself may go down to smaller units than these local areas - Census undercount estimation goes all the way down to blocks - but we are measuring heterogeneity only down to the scale allowed by P-12. For any particular variable of interest, each local area has a value that differs from the territory-wide value, the latter being an average of the former. The deviations from average are what we call heterogeneity, and it is heterogeneity that we are going to measure.

In our notation, within a given territory, for a given variable and demographic group:

$p_{i}$ is the true rate for all $n_{i}$ group members in the $i$ th of $L$ localities;

$p=\sum_{i} p_{i} / L$ is the arithmetic mean of the true local rates.

The quantity whose measurement is the goal of the study is the population-level "variance due to heterogeneity," the variance of the true local rates about their mean:

$$
H^{2}=\frac{1}{L} \sum_{i}\left(p_{i}-p\right)^{2}
$$

This quantity is important because it appears in formulas for estimation error. Let $\tilde{p}$ be an estimator 
of $p$. The mean squared error of estimation that results from using $\tilde{p}$ as the estimate of $p_{i}$ for all local areas as if they shared a common value is

$$
\frac{1}{L} \sum_{i}\left(p_{i}-\tilde{p}\right)^{2}=H^{2}+(p-\tilde{p})^{2}
$$

The first term on the right, $H^{2}$, represents errors due to heterogeneity resulting directly from attributing one common rate to local areas whose true rates vary. The second term represents errors due to bias and sampling variability in the territory-wide estimator $\tilde{p}$. The $H^{2}$-term only vanishes if the local areas are in fact homogeneous, so that all $p_{i}$ equal each other and hence equal $p$. Otherwise, $H^{2}$ is a contribution to error which cannot be reduced by increasing sample size.

Some fine points require mention. To begin with, $H^{2}$ is centered on $p$, in order to make straightforward the interpretation as a variance due to heterogeneity. Similarly, $p$ is the unweighted mean of the $p_{i}$. The $\mathrm{P}-12$ data were aggregated by a procedure that tends to equalize the counts of stratum members in local areas, so there is little numerical difference between weighted and unweighted means. Conceptually, however, the weighted mean is the natural target of a ratio estimator obtained from a numerator and denominator separately aggregated over $i$. If $\tilde{p}$ is such an estimator, then the term $(p-\tilde{p})^{2}$ in $\{2\}$ includes the squared difference between weighted and unweighted means, as well as a contribution from sampling error and from "ratio estimator bias," whose underlying source is again heterogeneity among the $p_{i}$. Ratio estimator bias enters through $p-\tilde{p}$, decreases with sample size, and is a minor part of the story compared to $H^{2}$ [Freedman, Stark and Wachter 2000].

We now discuss estimation of $H^{2}$ from the P-12 sample. Temporarily, we have fixed a territory and a variable, and are considering only persons in one demographic group. In our setup, the $i$ th superblock represents the $i$ th locality, so the number of superblocks coincides with the number of localities. Let

$\hat{p}_{i}$ be the rate for the $N_{i}$ sample persons in the $i$ th superblock;

$\hat{p}=\sum_{i} \hat{p}_{i} / L$ be the mean of these rates.

A naive estimator of $H^{2}$ would be

$$
\frac{1}{L} \sum_{i}\left(\hat{p}_{i}-\hat{p}\right)^{2}
$$

However, this estimator of variance due to heterogeneity is inflated by variance due to sampling. 
We therefore define $\hat{H}$ by the equation

$$
\hat{H}^{2}=\frac{1}{L} \sum_{i}\left(\hat{p}_{i}-\hat{p}\right)^{2}-\frac{1}{L}\left(1-\frac{1}{L}\right) \sum_{i} \frac{\hat{p}_{i}\left(1-\hat{p}_{i}\right)}{N_{i}-1} .
$$

The second term is an approximate correction for sampling variability in $\hat{p}_{i}$ and $\hat{p}$ [see Appendix].

\subsection{Variables and Strata}

Four variables are examined in our study:

(i) The multi-unit housing rate is the proportion of persons residing in multi-unit structures.

(ii) The non-mailback rate is the proportion of people who did not mail back their Census form, out of all people in the Census who were meant to mail it back.

(iii) The allocation rate is the proportion of persons with at least one of six key characteristics imputed. The six characteristics are relationship to householder, age, sex, race, Hispanic origin, and marital status.

(iv) The substitution rate is the proportion of persons whose whole record was imputed or "substituted" into the Census, typically in households from which no detailed information was obtained.

These variables provide a good variety of cases with which to examine local heterogeneity. They include one structural variable, one behavioral variable, and two measures of data completeness, all taking values between zero and one. They are four of the five main variables treated in the Census Bureau's P-12 Project Report [Kim 1991]: the fifth variable, the mail universe rate, is more narrowly administrative in character, and is not considered here.

Documentation of the P-12 data set is to be found in [Thompson 1990, U.S. Bureau of the Census 1990, Bateman 1991, Kim 1991]. The sample is a stratified cluster sample selected using essentially the same design as the Census Bureau's 1990 Post-Enumeration Survey (PES) but with 116,619 block clusters in place of 5293. These clusters include 204,394 blocks compared to 12,964 blocks for the PES.

The stratification is the one proposed for adjusting the 1990 Census. The population is divided into 116 PSGs ("Post-Stratum Groups") defined in part by geography and in part by demography. The geographical classification is based on census division ( 9 areas) and place-type (7 types). The demographic breakdown is by race-ethnicity (4 categories), and renter-owner status (2 groups). In principle, there could be $9 \times 7 \times 4 \times 2=504$ PSGs, but smaller ones are collapsed and the largest urban areas are treated differently. In total, there are 116 PSGs: a list can be found in Table A.1 of Hogan (1993). We exclude the PSG for Indians living on reservations, and deal with the remaining 
115; we also exclude the so-called "residual population" not surveyed by the PES. Each PSG is broken down by 6 age groups and 2 sexes into 12 "post-strata," so we have $115 \times 12=1380$ post-strata to consider.

Each post-stratum is defined in part by demography (race-ethnicity, renter-owner, age, sex) and in part by geography (census division, place-type). Three examples give the flavor of the post-strata:

(i) Non-minority females age 0-9 living in a central city in New England.

(ii) Black males age 10-19 living in rental units in a central city in a large metropolitan area in the South Atlantic division (Florida, Georgia, and so forth).

(iii) Black and hispanic females age 60 and over in New England, living either in a central city or in a metropolitan area but not in its central city

The geography is the "territory" associated with the post-stratum, and the demography can be considered as providing the stratification within which small-area estimation takes place. In post-stratum (i), for instance, the territory is central cities in New England; small-area estimation would be uniform within the part of this territory inhabited by the demographic group consisting of non-minority females age 0-9. With post-stratum (ii), the territory consists of central cities in large metropolitan areas in the South Atlantic division, and the demographic group consists of black males age 10-19 living in rental units. Thus, the boundaries of the territories depend on the poststratum, and so will the dissection of each territory into local areas. For groups whose members are numerous, high resolution is possible and the local areas are small. For groups whose members are few and far between, the local areas are extended.

As noted above, the specification of territories, and localities within a territory, is data-dependent. More specifically, the records in P-12 correspond to unique - and non-overlapping - intersections of post-strata and superblocks. These records were built up from more basic information for each post-stratum and sample block. The algorithm used to build up the records required that for any given post-stratum, a P-12 record must have at least ten post-stratum members; the corresponding geography must span whole Census block clusters and must not cross state lines. (These three constraints could not always be satisfied, and we eliminated some 2000 exceptional records.) Given a post-stratum, a "superblock" is the collection of block clusters put together during the construction of a record; there is one superblock per record. There is one locality per superblock, consisting of all the block clusters in the population corresponding to the one block cluster in the sample. Due to the sample design, this informal idea can be made fairly precise [see Appendix].

The total U.S. population is around 250 million. Our P-12 dataset has about 12,000,000 people, and we think of it as a 1-in-20 sample; in reality, the sampling fraction varies from one part of 
the country to another. There were 750,000 records, so the average number of sample persons in a record is $12,000,000 / 750,000=16$ : given a typical post-stratum and superblock, about 16 members of the post-stratum will be found in the superblock. This corresponds to roughly 300 post-stratum members per locality, since each sample person represents some 20 people. The algorithm used to construct the P-12 records tends to equalize the local-area counts.

There are 115 PSGs; these do not overlap, and their average size is around 250 million/115 or 2.2 million people. Each PSG is defined by a combination of geography - the "territory" - and demography. The territories will have a population that is several times larger than the PSG: 5-10 million people is a representative range, so there must be several hundred localities per territory. We estimate an average of 6 blocks per superblock, hence, 120 blocks per locality, with 6,000 persons in all demographic groups combined - although this is only an order-of-magnitude calculation. We have done the P-12 aggregation ourselves on the "Berkeley subset" of census data [see Appendix], and those simulations suggest a population of 10,000 per locality. In the end, we think most localities will have populations in the range 2,500-25,000.

The geometry is confusing at first. The superblocks and localities associated with any particular post-stratum do not overlap. As we move from one post-stratum to another within the same PSG, the territory remains the same - but due to the aggregation procedure, the superblocks change and new superblocks overlap the old. (Similar statements apply to the localities.) As we move from one PSG to another, the territories change and overlap: compare post-strata (i) and (iii) above.

Although details are complicated, the basic picture is straightforward. There are two scales which govern any measurement of heterogeneity. Variability occurs within some big unit, across some small units. Here the big unit is a territory encompassing something like 7 million people. The small units are local areas encompassing some 10,000 people, with about 300 people in each of 30 demographic groups. On these scales, P-12 allows estimates of residual heterogeneity after stratification by demographic group. The measurement of heterogeneity within post-strata across the superblocks of P-12 is relatively unambiguous; tying the results to more familiar geographical units must be more tentative, due to the complexities of the P-12 data structure.

\subsection{Results}

Estimates of residual heterogeneity from P-12 are shown in Table 1, along with related values for comparison. The four columns correspond to the four outcome variables in our study. The values shown for $\hat{H}$ and for sampling standard error are root-mean-square (RMS) values calculated over all post-strata. We report $\hat{H}$ rather than $\hat{H}^{2}$ to make units and scale more easily understandable. 
Table 1: Measures of Heterogeneity and Comparative Values

\begin{tabular}{lcccc}
\hline & $\begin{array}{c}\text { Multi-unit } \\
\text { housing }\end{array}$ & $\begin{array}{c}\text { Non- } \\
\text { mailback }\end{array}$ & $\begin{array}{c}\text { Alloca- } \\
\text { tions }\end{array}$ & $\begin{array}{c}\text { Substi- } \\
\text { tutions }\end{array}$ \\
\hline $\begin{array}{l}\text { Measures of Heterogeneity } \\
\hat{H} \text { for local areas }\end{array}$ & $22.3 \%$ & $10.7 \%$ & $7.1 \%$ & $2.3 \%$ \\
$\quad$ Standard error & $0.7 \%$ & $0.3 \%$ & $0.2 \%$ & $0.2 \%$ \\
$\hat{H}$ for states & $10.4 \%$ & $4.3 \%$ & $2.9 \%$ & $0.6 \%$ \\
Values for Comparison & & & & \\
Standard deviation of & & & & \\
$\hat{p}$ across post-strata & $23.7 \%$ & $12.0 \%$ & $8.0 \%$ & $0.7 \%$ \\
Mean of $\hat{p}$ across post-strata & $28.6 \%$ & $29.7 \%$ & $19.7 \%$ & $1.1 \%$ \\
Sampling standard errors of $\hat{p}$ & & & & \\
$\quad$ Low estimate & $2.6 \%$ & $3.0 \%$ & $2.7 \%$ & $0.7 \%$ \\
$\quad$ High estimate & $6.1 \%$ & $4.2 \%$ & $3.4 \%$ & $1.0 \%$ \\
\hline
\end{tabular}

The first row of Table 1 shows $\hat{H}$ s for local areas in P-12. The first entry is $22.3 \%$, signifying that within post-strata, the local area-to-area differences in the rates of multi-unit housing are on the order of $22.3 \%$. In other words, ascribing the overall rate of multi-unit housing to the local areas within a territory incurs an RMS error due to heterogeneity of $22.3 \%$, even after controlling for the geographic and demographic variables in the post-stratification. This outcome reveals a remarkable degree of diversity in the clustering of apartment buildings and multiple-family houses. The other entries range from $10.7 \%$ for the non-mailback rate down to $2.3 \%$ for the Census substitution rate. The calculation of the standard errors is described below [see Appendix]; these are plausible upper bounds.

If people belonging to the same post-stratum shared the same rates, wherever they resided, the values of $H^{2}$ would all be estimates of zero. Though $H^{2}$ is estimating a non-negative quantity, its sample values are not constrained to be non-negative. None of the 1380 post-strata have negative estimates for multi-unit housing or non-mailbacks; five do for allocations and 50 for substitutions. The small standard errors and the rarity of negatives both indicate the strong statistical significance of the observed heterogeneity, thanks to the large sample size of P-12.

Our formula for $\hat{H}$ can be applied to measure state-to-state heterogeneity by letting $i$ in the 
definition range over the 50 states plus the District of Columbia. The third row of Table 1 shows the state-level RMS values of $\hat{H}$ across the 1224 post-strata that intersect more than one state. We see that $\hat{H}$ for multi-unit housing only falls to a little less than half its local-level value at this much larger level of aggregation. For substitutions, $\hat{H}$ is still one-fourth of its local level. Heterogeneity is not simply produced by small-scale flutters in concentrations: if it were, heterogeneity would average out at larger scales like states. The values for states in Table 1 are generally only a bit smaller than the comparable values for states in Table 5 of [Freedman and Wachter 1994], where a coarser 357-fold post-stratification is used; the value for multi-unit housing is actually bigger. This suggests that the measures of heterogeneity are somewhat robust to moderate changes in the post-stratification. Put another way, refining the stratification may not yield much reduction in heterogeneity.

The practical significance of the levels of heterogeneity indicated by the first row of Table 1 may be judged by various standards of comparison. One natural comparison is with the standard deviation of the post-stratum mean rates $\hat{p}$ across post-strata, shown in the fourth row of Table 1 . This standard deviation suggests itself when one thinks of the values for, say, the multi-unit housing rate as entries in a two-way table whose rows are superblocks and whose columns are post-strata. The index $\hat{H}$ then measures the residual variability after controlling for column effects, and the standard deviation over post-strata measures the variability "explained" by the column effects. Table 1 shows that the residual variability is roughly as large as the explained variability. That is true for the first three variables. For the fourth, substitutions, the residual variability is three times as large. For comparable data at the state level and an algebraic treatment that dispels the air of paradox, see [Freedman and Wachter 1994].

The levels of $\hat{H}$ may also be judged by comparison with the sampling standard errors for the post-stratum mean rates $\hat{p}$. The last two rows of Table 1 show a low and a high estimate of sampling standard error based on a sample of the size of the PES, the post-enumeration survey for 1990. The derivation of our two illustrative estimates of standard error is explained below [see Appendix]. It turns out that the local heterogeneity measured by $\hat{H}$ is much larger than the sampling standard error with samples of this size. Even at the state level, heterogeneity is comparable to the sampling standard errors for $\hat{p}$. Obviously, heterogeneity cannot be taken to be negligible in comparison with sampling variability in any settings like the ones considered here. The numbers in Table 1 are based on averaging post-strata; however, examination of scatterplots (not presented here) indicates that the conclusions hold for practically all individual post-strata.

For variables like ours, taking values between zero and one, the mean of the variable imposes a constraint on the variance due to heterogeneity. Hence we expect the levels of $\hat{H}$ to be strongly 
influenced by the post-stratum means $\hat{p}$. For instance, only $1.1 \%$ of person-records on average are Census substitutions while $28.6 \%$ correspond to people in multi-unit housing; the corresponding $\hat{H}$ s are $2.3 \%$ and $22.3 \%$. Post-stratum by post-stratum plots of $\hat{H}^{2}$ versus $\hat{p}$, not given here, show $\hat{H}$ tends to vary like a fraction of $\sqrt{\hat{p}(1-\hat{p})}$. We call the ratio $\hat{H} / \sqrt{\hat{p}(1-\hat{p})}$ the "max-fraction." Its median value across the 1380 post-strata is roughly $1 / 2$ for multi-unit housing, 1/4 for the non-mailback rate, 1/6 for the allocation rate, and 1/5 for the substitution rate.

The "max-fraction" is given its name for the following reason. The maximum amount of heterogeneity in (say) multi-unit housing is achieved by an all-or-nothing arrangement where a proportion $p$ of the local areas have nothing but apartments and the remaining $1-p$ of the local areas have nothing but single-family houses. Under this arrangement, $H$ takes on the maximum value consistent with an overall mean of $p$, namely $\sqrt{p(1-p)}$. Under any less heterogeneous arrangement, $H$ takes on some fraction of its maximum. The max-fraction, a sample-based estimate of the population-level quantity, is a measure of heterogeneity standardized for the level of $\hat{p}$. Since mean max-fractions are sensitive to a handful of outliers, medians may be more descriptive. For multi-unit housing, $\hat{H}$ is over half the maximum possible level. By this standardized measure, the allocation rate shows the least heterogeneity and the multi-unit housing rate the most.

Table 2: Local-Level $\hat{H}$ for Groups of Strata

Root Mean Square Values across Post-Strata

\begin{tabular}{lcccc}
\hline & $\begin{array}{c}\text { Multi-unit } \\
\text { housing }\end{array}$ & $\begin{array}{c}\text { Non- } \\
\text { mailback }\end{array}$ & $\begin{array}{c}\text { Alloca- } \\
\text { tions }\end{array}$ & $\begin{array}{c}\text { Substi- } \\
\text { tutions }\end{array}$ \\
\hline Male strata & $22.0 \%$ & $11.0 \%$ & $7.3 \%$ & $2.3 \%$ \\
Female strata & $22.6 \%$ & $10.4 \%$ & $6.9 \%$ & $2.3 \%$ \\
Ages 0 through 9 & $23.2 \%$ & $13.2 \%$ & $8.5 \%$ & $2.6 \%$ \\
Ages 20 through 29 & $25.4 \%$ & $11.5 \%$ & $6.9 \%$ & $2.4 \%$ \\
Owner strata & $17.3 \%$ & $8.7 \%$ & $6.6 \%$ & $2.0 \%$ \\
Mixed strata & $22.2 \%$ & $11.0 \%$ & $6.9 \%$ & $2.2 \%$ \\
Renter strata & $26.6 \%$ & $11.2 \%$ & $8.3 \%$ & $3.0 \%$ \\
\hline
\end{tabular}

Table 2 presents estimated $\hat{H}$ s for various groups of strata; only two age-ranges are shown. The differences are modest: for instance, values for males and females are very close. The higher $\hat{H}$ s are generally associated with higher mean $\hat{p}$ s. The post-strata which mix renters and owners 
together do not show more heterogeneity than the post-strata which separate out renters: the latter strata have the higher mean $\hat{p}$ s.

Breakdowns by groups, like those in Table 2, show that heterogeneity is pervasive. Heterogeneity is not concentrated among post-strata of any particular type. Strata which mix groups like owners and renters produce similar levels of heterogeneity as strata which separate them. That outcome is further evidence that dependence on the details of post-stratification is not severe. By contrast, heterogeneity would be expected to vary with the geographical resolution. Table 3 shows $\hat{H}$ s from studies with different levels of resolution; the variable used is the allocation rate.

Table 3: Dependence of $\hat{H}$ on Geographical Level for Allocations

\begin{tabular}{lccc}
\hline Geography & Data set & Post-strata & $\hat{H}$ \\
\hline States within U.S. & P-12 & 1392 & $2.9 \%$ \\
States within U.S. & 1990 Census & 357 & $3.9 \%$ \\
PUMAs within Oregon & 1990 PUMS & 120 & $6.6 \%$ \\
Localities within territories & P-12 & 1392 & $7.1 \%$ \\
\hline
\end{tabular}

Sources: Table 1 in the present study for lines 1 and 4; [Freedman and Wachter 1994] for line 2; [Rhyne 1999] for line 3.

Table 3 has results for "Public Use Microdata Areas" (PUMAs), which are aggregations of cities and counties into areas each of which contains at least 100,000 people. The results are due to Marcey-Jo Rhyne and are quoted by permission. Her post-stratification for the PUMAs follows the one used in the 1990 PES, to the extent feasible: no distinctions of place-type can be made; renters are distinguished from owners in all cases, as are blacks, non-black hispanics, Asian and Pacific Islanders, and whites and others. She looked only at allocations. It is interesting that the heterogeneity across the relatively large PUMA units within one state is nearly as high as the heterogeneity across the much smaller local areas within larger territorial groupings. 


\section{Applications to Census Undercount Estimation}

The results of Section 2 provide guidance about the likely size of errors due to heterogeneity in the Census Bureau's small-area estimates of undercounts from the 1990 PES. They provide such guidance to the extent that the P-12 variables provide meaningful analogues to undercounts with respect to place-to-place variability, and to the extent that P-12 resembles the PES in sample design and post-stratification. The P-12 variables were chosen specifically to provide such analogues. Like undercounts, they are Census coverage indicators, and the Census Bureau goes so far as to call them "proxies" or "surrogates" for undercount. The P-12 sample design was chosen to be essentially the same as that for the PES, and the post-stratifications are identical. These considerations all support the idea of taking P-12 as a guide to the effects of heterogeneity on 1990 undercount estimates.

On the other hand, there is no direct validation of the posited similarity between P-12 variables and undercounts. The main available comparisons are in terms of overall levels and indices of dispersion. These are presented in this section. It turns out that undercounts fall well within the range of alternatives spanned by the four P-12 variables, but no single P-12 variable is a close match in both level and dispersion.

Net undercounts can be negative (when there is an overcount) but the P-12 variables are always non-negative. This is an important difference which weakens the analogy. The net undercount is approximately equal to the difference between two non-negative variables, the rates of "gross omissions" (e.g., missed persons) and "erroneous enumerations" (e.g., duplicates or fabrications). The P-12 variables may be better analogues for these two components of undercount than for their difference, but the overall picture is complicated by the correlations between gross omissions and erroneous enumerations which extend within post-strata all the way down to Census blocks.

Information on levels and indices of dispersion for undercount variables are shown in Table 4. They are to be compared to the corresponding rows for P-12 variables in Table 1. In Table 4, following common Bureau practice, centered adjustment factors are used in place of undercount rates. The centered adjustment factor for any unit is calculated by taking the estimated true count, dividing by the Census count, and subtracting one. The centered adjustment factor is close to the undercount rate itself. The first column in Table 4 pertains to the Bureau's "smoothed" adjustment factors, the factors actually used for the Bureau's calculation of adjusted counts. The second column pertains to the "raw" adjustment factors. These are dual-system estimates from PES data, calculated post-stratum by post-stratum. The raw factors were transformed into the smoothed factors by an empirical Bayes smoothing algorithm [Freedman et al. 1993]. The final two columns 
pertain to the gross omission and erroneous enumeration rates. Neither Table 4 nor Table 1 is weighted for post-stratum size.

Table 4: Comparative Values for PES Adjustment Factors

\begin{tabular}{lcccc}
\hline & $\begin{array}{c}\text { Smoothed } \\
\text { Factors }\end{array}$ & $\begin{array}{c}\text { Raw } \\
\text { Factors }\end{array}$ & $\begin{array}{c}\text { Gross } \\
\text { Omissions }\end{array}$ & $\begin{array}{c}\text { Erroneous } \\
\text { Enumerations }\end{array}$ \\
\hline $\begin{array}{l}\text { Standard deviation } \\
\quad \text { across post-strata }\end{array}$ & $4.1 \%$ & $7.0 \%$ & $6.7 \%$ & $4.5 \%$ \\
$\begin{array}{l}\text { Mean of centered factors } \\
\text { RMS of Bureau's }\end{array}$ & $2.8 \%$ & $2.9 \%$ & $9.7 \%$ & $6.4 \%$ \\
$\quad$ estimated standard errors & $2.0 \%$ & $5.9 \%$ & unknown & unknown \\
\hline
\end{tabular}

The level and dispersion of a variable undoubtedly affect the numerical values of $\hat{H}$ for the variable, so the comparisons between Table 1 and Table 4 are important indicators of the relevance of P-12 to undercounts. With one exception, we see that all entries in Table 4 fall between the corresponding values for substitutions and for allocations in Table 1. The exception is the 5.9\% sampling standard error for the raw factors, which falls above the standard error for allocations and just below the high estimate of standard error for multi-unit housing. Thus, in terms of the quantities shown in Table 4, the P-12 variables do span the relevant range, but none matches on all dimensions.

An important conclusion is suggested by comparing the figure of $2.0 \%$ in the lower left of Table 4 with the figures in the first row of Table 1 . The $2.0 \%$ is the RMS of the Bureau's estimates of sampling standard error for its smoothed adjustment factors, and it is lower than any of the RMS values of $\hat{H}$ for local areas in Table 1 . If the P-12 variables are at all valid analogues, then the estimated PES sampling variances are evidently dominated by the variance due to heterogeneity measured by $\hat{H}^{2}$. Sampling variance is the contribution to error which the Bureau did include in its error margins for adjusted local counts [U.S. Bureau of the Census 1991]. Variance due to heterogeneity is one of the contributions it did not include. The data here suggest that what was left out is more important than what was put in.

It is likely that some part of the true contribution from sampling variability was also left out. 
The $2.0 \%$ figure for sampling standard deviation is believed to be a considerable underestimate [Fay and Thompson 1993, Freedman et al. 1993]. In principle, sampling variance can be traded off against variance due to heterogeneity by adopting a coarser or finer post-stratification. But the variances due to heterogeneity implied by Table 1 are so large that the leeway for such tradeoffs appears rather slight.

The particular use we are making of P-12, with our concentration on heterogeneity alone and our direct calculation of $\hat{H}$ within post-strata, avoids certain difficulties which would confront more ambitious uses. We are not calculating measures of overall error for local counts or shares. Thus we are not engaged in assessing the augmentations or cancellations of error that take place when the positive or negative estimated adjustments for different post-strata in the same local area are added together to yield the total estimate for the area. We cannot do so with P-12, because P-12 superblocks for different post-strata do not coincide. Heterogeneity implies error both in Census counts and in adjusted counts, and the balance between these errors appears to be a delicate function of patterns of cancellation when post-stratum contributions are summed. We are also not engaged in studying the interaction between errors in local counts due to heterogeneity and errors at all levels due to bias in post-stratum-wide adjustment factors. We are studying errors in an idealized, bias-free setting. This setting would correspond to a PES in which the post-stratumwide adjustment factors were known perfectly. Our counterparts of post-stratum-wide factors, that is, our $\hat{p}$ s, are unbiased.

The post-stratum-wide adjustment factors in the real PES are known to be biased. There is, of course, some ratio-estimator bias. That is a side-effect of heterogeneity, and should be distinguished from the heterogeneity studied in this report, which affects estimated rates for local areas within post-strata. There are other, more important, biases in the adjustment factors estimated by the PES. Attempts have been made to measure some of these by quality-control and followup studies, but only at the level of large aggregations of post-strata. Biases are quantified in [Breiman 1994] and in Table 15 of the Census Bureau's P-16 Project Report. Unfortunately, this crucial table is omitted from the published version [Mulry and Spencer 1993]. There is also unmeasured "correlation bias" resulting from the tendency for people missed by the Census to be more likely to be missed by the PES estimates. Essentially nothing is known about how the measured biases are distributed among the post-strata, and even less about the size and distribution of correlation bias. Thus there is not yet a basis on which definitive assessments of the relative accuracy of adjusted and unadjusted counts for local areas could be made - unless some rather heroic assumptions are to be imposed on the data. For recent reviews, see [Brown et al. 1999, Wachter and Freedman 2000], but those findings seem to be disputed in [Prewitt 2000]. 
In short, at the local level, what can be made are assessments of components of error like heterogeneity, not assessments of relative accuracy. To strengthen the assessments, it would be valuable to relate P-12 more closely to the PES. The Census Bureau (as far as we can tell) has not released data sufficient to calculate place-to-place correlations between the variables studied here and undercounts. In principle, substitutions, allocations, non-mailback rates, and multi-unit housing rates exist along with undercount estimates for the 5392 PES block clusters. Even more relevant than such cross-correlations would be autocorrelation functions for the variables, calculated as functions of physical or notional distance between areas. The PES sample size is small for this purpose, but some insights could be gleaned. At present, the correlations that can be computed are those that are least relevant - across post-strata. Smoothed adjustment factors correlate 0.60 with non-mailback rates, 0.23 with multi-unit housing rates, 0.18 with substitution rates, and 0.07 with allocation rates, across post-strata. Substitution and allocation rates correlate 0.61 with each other.

The PES sample is too small to give estimates of heterogeneity of the precision obtained from P-12. At the local level, the data for an $\hat{H}$ calculation are not available to us at all for most poststrata. At the state level, using weighted data by post-stratum and calculating as if the sampling weights were uniform within post-strata, we find RMS values for $\hat{H}$ for state-to-state heterogeneity of $10 \%$ for gross omissions and $7 \%$ for erroneous enumerations. These figures fall near the upper end of the RMS state-level $\hat{H}$ values in Table 1. The PES estimates for single post-strata are unstable to the extent that about $25 \%$ of post-strata come out with negative estimated values of $\hat{H}^{2}$. The RMS values over all 1380 post-strata are bound to be more stable, and the figures suggest that heterogeneity in components of undercount is at least as great as heterogeneity in the P-12 variables. 


\section{Prior Literature}

Notwithstanding the large literature on methods for small-area estimation, there have been comparatively few evaluation studies and even fewer attempts to quantify errors due to heterogeneity. The literature on methods, building on [Deming 1948], is summarized by [Purcell and Kish 1979, Platek et al. 1987, Ghosh and Rao 1994]. Uniform ratio estimators like the ones considered in this study are the oldest and most widespread of all small-area estimators. They are sometimes themselves called "synthetic estimators," though that name, coined in [National Center for Health Statistics 1968], is more properly applied when such estimators have been summed up within areas over strata or groups.

Parametric evaluations based on variance-component models have been applied and studied [Battese, Harter and Fuller 1988, Prasad and Rao 1990]. For that work, unlike P-12 and the PES, each of the small areas for which estimates are needed contains sampled units; parameters governing heterogeneity are identifiable without the presence of a census or evaluation sample like $\mathrm{P}-12$. When direct comparisons and parametric estimates are not feasible, evaluations of small-area estimates generally take the form of sensitivity analyses and simulation studies.

The literature on evaluation of small-area estimates tends to focus, like our report, on problems of census adjustment. There is a simulation study of synthetic estimation using two demographic groups [Schirm and Preston 1987]. The areas are states plus the District of Columbia; the variable is the 1980 net Census undercounts. Lacking information about levels of heterogeneity of the kind given in the present report, a stylized model is used. Group-specific state effects are assumed to be independent and identically distributed lognormal variables, with variances set to levels loosely suggested by Census Bureau work on 1970 undercounts.

A form of evaluation that has come to be called "artificial population analysis" has been pursued with 1980 Census data [Isaki et al. 1987]. Related, as yet unpublished, work by Census Bureau staff has been conducted with 1990 data. Both "across-the-board" (unstratified uniform ratio estimates) and synthetic estimates have been studied, also with 1980 data [Wolter and Causey 1991]. The areas are states, counties, and 1980 Census enumeration districts (with typical populations of a thousand or so). The variable under study is the Census substitution rate (also studied in P-12), rescaled within strata to match certain 1980 national net undercount estimates. The "across the board" studies use six strata defined by place-type within New England. The synthetic studies use 24 strata defined by age, sex, and race within the whole United States. Results are presented in terms of several aggregate "measures of closeness" for adjusted versus unadjusted values. A discussion of these studies can be found in [Freedman and Navidi 1992]. 
Using block-level data for components of undercount from the 1990 PES, within-group heterogeneity across blocks has been compared to within-block heterogeneity across groups [Hengartner and Speed 1993]. In a study of Australian unemployment rates, small-area estimates are evaluated by a direct comparison with tabulations from a contemporaneous census [Feeney 1987]. Our work with P-12 is an approximate version of this direct strategy, in which an extra-large sample from the census plays the role of the census itself.

The Bureau has analyzed the P-12 data, concentrating on the statistical significance of state-tostate heterogeneity [Kim 1991, Kim, Blodgett and Zaslavsky 1993]. Several approaches were used, including log-linear modeling of the P-12 variables, estimating state effects separately for poststratum groups. The test statistics measure excess heterogeneity from state to state after dividing out the observed heterogeneity from local area to local area. This confounds the effects of local heterogeneity with the effects of sample design. Given the high level of local heterogeneity, this analytic strategy has little power for detecting state-to-state heterogeneity.

Methods like those of the present study have been applied to measure state-to-state heterogeneity in six Census coverage indicators including the four studied here [Freedman and Wachter 1994]. That work is based on the whole Census, not on a sample like P-12, and it uses a post-stratification with 357 strata instead of the 1392 used here. For various state-by-state tallies, the impact of heterogeneity on loss-function analyses is quantified. The impact of other omitted or underestimated sources of error on the Census Bureau's loss function analyses for 1990 has been reviewed [Freedman et al. 1994].

Previous investigators have detected residual heterogeneity in probabilities of enumeration by the 1990 census [Alho et al. 1993]. The investigation focused on minorities in central cities across the four census regions, and used logistic regression. One explanatory variable was the multi-unit housing rate, which turned out to be strongly associated with capture in the census, at least in two regions. Substitutions and allocations were excluded from the model, but were also strongly associated with capture in the census. Overall, the impact of heterogeneity is estimated as being roughly half the size of the net undercount. Geographic heterogeneity at state or substate levels was not explicitly represented: the modeling was done at the level of individuals within broad groups of post strata, some explanatory variables being defined at the post-stratum level.

Many observers favor census adjustment; illustrative citations are [Schirm and Preston 1987, Ericksen, Kadane and Tukey 1989, Wolter and Causey 1991, Mulry and Spencer 1993, Zaslavsky 1993, Belin and Rolph 1994, Steffey and Bradburn 1994, Anderson and Fienberg 1999, Cohen, White and Rust 1999, Prewitt 2000]. Other observers find that census adjustment would introduce more error than it removes [Freedman and Navidi 1992, Hengartner and Speed 1993, Freedman et 
al. 1993, Breiman 1994, Freedman et al. 1994, Freedman and Wachter 1994, Brown et al. 1999, Darga 1999, Wachter and Freedman 2000, Skerry 2000, Stark 2000]. There are a priori reasons to favor adjustment; on the other hand, there are substantial biases in estimated adjustment factors, and heterogeneity is pervasive. What is difficult to determine from available data is the extent to which biases reinforce each other or cancel, even at the state level; the bottom-line impact of heterogeneity on accuracy is another major issue.

On the wider question of amounts of heterogeneity to be expected for variables of various kinds at local levels, we are aware of no systematic empirical studies. The analysis of local Census data as a field of study is summed up by [Myers 1992]. Better empirical knowledge about geographical heterogeneity in demographic behavior is important not only for small-area estimation but also for the modeling of long-term demographic change. Parish-to-parish variability in English historical data has been analyzed [Wachter 1992]. Stochastic demographic models which recognize geographic levels of randomness in human population processes are the eventual goal. 


\section{Conclusions}

In summary, we have introduced a direct measure of heterogeneity, $\hat{H}$, and used it to measure heterogeneity from local area to local area for four variables related to Census coverage. The source of the data is the Census Bureau's P-12 sample from the 1990 U.S. Census. The heterogeneity we have measured is residual heterogeneity after stratification by age, sex, race and ethnicity, renterowner status, place-type and broad geographical division of the country. The local areas are units with total populations around 10,000. We find that the area-to-area variance within strata - reflecting geographical heterogeneity - is roughly comparable to the variance from stratum to stratum, even for this fine a stratification.

The variables examined in this study are believed by the Census Bureau to offer meaningful analogues to Census undercount. If this is true, then our results imply that errors due to heterogeneity from local area to local area dominate errors due to sampling variability in the small-area ratio estimation step of the Bureau's undercount estimates. The errors treated as negligible in the calculation of error margins are larger than the errors included in the calculation. It follows that the Census Bureau's published margins of error for adjusted Census counts for local areas are likely to be substantial underestimates.

For stratified small-area ratio estimation, our results suggest that the popular "default option" of treating residual heterogeneity as negligible is a serious mistake. When direct measures of error due to heterogeneity are unavailable, a better default option would be to treat residual heterogeneity as being on a par with the variance explained by the stratification factors.

Variables like the P-12 rates can typically vary by 5,10 or 20 percentage points from local area to local area, even for people of the same age, sex, race, and ethnicity living in communities of the same general size in the same broad areas of the country. In the absence of direct evidence to the contrary, simulation studies of the efficacy of small-area estimation should allow for substantial local heterogeneity. "Diversity" is a byword in America's political vocabulary. Diversity is certainly the rule, when one looks from place to place across America with the Census Bureau's 1990 P-12 sample. 


\section{Acknowledgements}

We thank the Donner Foundation for its financial support. The analysis has been carried out by us with substantial assistance from Daniel Coster, Richard Cutler, Charles Everett, and Mark Hansen. We are grateful to the U.S. Census Bureau for making the P-12 data available and for many helpful explanations. We have served as consultants to the Freshpond Institute and as expert witnesses for the government in litigation over proposed adjustments of the 1980 and 1990 censuses. 


\section{References}

Alho, J.M., M.H. Mulry, K. Wurdeman, and J. Kim (1993). "Estimating Heterogeneity in the Probabilities of Enumeration for Dual System Estimation." Journal of the American Statistical Association, 88: 1130-36.

Anderson, M. and S.E. Fienberg (1999). Who Counts? The Politics of Census-Taking in Contemporary America. New York: Russell Sage Foundation.

Bateman, D. (1991). "Specifications for Data Analysis of PES Project P12 Data." Memorandum Series \#N-3, U.S. Bureau of the Census, Washington, D.C.

Battese, G., R. Harter, and W.A. Fuller (1988). "An Error-Components Model for Prediction of County Crop Areas Using Survey and Satellite Data." Journal of the American Statistical Association, 83: 28-36.

Belin, T.R. and J.E. Rolph (1994). “Can We Reach Consensus on Census Adjustment?” Statistical Science, 9: 486-508 (with discussion).

Breiman, L. (1994). “The 1990 Census Adjustment: Undercount or Bad Data?" Statistical Science, 9: 458-475.

Brown, L.D., M.L. Eaton, D.A. Freedman, S.P. Klein, R.A. Olshen, K.W. Wachter, M.T. Wells, and D. Ylvisaker (1999). "Statistical Controversies in Census 2000.” Jurimetrics, 39: 347-375.

Cohen, M.L., A.A. White, and K.F. Rust, editors (1999). Measuring a Changing Nation: Modern Methods for the 2000 Census. Washington, D.C.: National Academy Press.

Darga, K. (1999). Sampling and the Census, Washington, D.C.: The AEI Press.

Deming, W.E. (1948). Statistical Adjustment of Data. New York: John Wiley and Sons.

Ericksen, E.P., J.B. Kadane, and J.W. Tukey (1989). “Adjusting the 1980 Census of Population and Housing." Journal of the American Statistical Association, 84: 927-944.

Fay, R. and J. Thompson (1993). "The 1990 Post Enumeration Survey: Statistical Lessons in Hindsight." Proceedings of the 1993 Annual Research Conference of the U.S. Bureau of the Census. Washington, D.C.: 71-91.

Feeney, G.A. (1987). “The Estimation of the Number of Unemployed at the Small Area Level." In Platek, R., J. Rao, C. Sarndal, and M. Singh, editors (1987). Small Area Statistics. New York: John Wiley and Sons.

Freedman, D.A. and W.C. Navidi (1992). "Should We Have Adjusted the U.S. Census of 1980?" Survey Methodology, 18: 3-74. 
Freedman, D.A., P.B. Stark, and K.W. Wachter (2000). "A Probability Model for Census Adjustment." Mathematical Population Studies, to appear. Technical Report 557, Department of Statistics, U.C. Berkeley.

Freedman, D.A. and K.W. Wachter (1994). "Heterogeneity and Census Adjustment for the InterCensal Base.” Statistical Science, 9: 476-485, 527-537.

Freedman, D.A., K.W. Wachter, D. Coster, R.C. Cutler, and S.P. Klein (1993). "Adjusting the Census of 1990: The Smoothing Model.” Evaluation Review, 17: 371-443.

Freedman, D.A., K.W. Wachter, R.C. Cutler, and S.P. Klein (1994). “Adjusting the U.S. Census of 1990: Loss Functions." Evaluation Review, 18: 243-280.

Ghosh, M. and J.N.K. Rao (1994). “Small Area Estimation: An Appraisal.” Statistical Science, 9: 55-76.

Hengartner, N. and T.P. Speed (1993). "Assessing Between-Block Heterogeneity Within the Poststrata of the 1990 Post-Enumeration Survey." Journal of the American Statistical Association, 88: 1119-1125.

Hogan, H. (1993). “The 1990 Post-Enumeration Survey: Operations and Results.” Journal of the American Statistical Association, 88: 1047-1061.

Isaki, C., G. Diffendal, and L. Schultz (1987). "Report on Statistical Synthetic Estimation for Small Areas." Technical Report 87-20, U.S. Bureau of the Census, Washington, D.C.

Kim, J. (1991). “P-12 Project Report.” U.S. Bureau of the Census, Washington, D.C.

Kim, J., R. Blodgett, and A. Zaslavsky (1993). "Evaluation of the Synthetic Assumption in the 1990 Post-Enumeration Survey." Technical Report, U.S. Bureau of the Census, Washington, D.C.

Le Bras, Hervé (1993). La planète au village. Paris: Editions de l'Aube.

Mulry, M.H. and B.D. Spencer (1993). "Accuracy of 1990 Census and Undercount Adjustments.” Journal of the American Statistical Association, 88: 1080-1091.

Myers, Dowell (1992). Analysis with Local Census Data. Boston: Academic Press.

National Center for Health Statistics (1968). Synthetic Estimates of Disability. Public Health Service Publication No. 759, Washington, D.C.

Platek, R., J. Rao, C. Sarndal, and M. Singh, editors (1987). Small Area Statistics. New York: John Wiley and Sons.

Prasad, N.G.N. and J. Rao (1990). "The Estimation of the Mean Squared Error of Small-Area Estimators." Journal of the American Statistical Association, 85: 163-171. 
Prewitt, K. (2000). "Accuracy and Coverage Evaluation; Statement of the Feasibility of Using Statistical Methods To Improve the Accuracy of the Census 2000.” Federal Register 65, Tuesday 20 June 2000: 38374-38398.

Purcell, N.P. and L. Kish (1979). “Estimation for Small Domains.” Biometrics, 35: 365-384.

Rhyne, M. J. (1999). "Measuring Heterogeneity from Public Use Microdata Samples.” Senior Thesis, Department of Statistics, U.C. Berkeley.

Schirm, A. and S. Preston (1987). "Census Undercount Adjustment and the Quality of Geographic Population Distributions.” Journal of the American Statistical Association, 82: 965-983.

Skerry, P. (2000). Counting on the Census. Washington, D. C.: Brookings.

Stark, P.B. (2000). “The 1990 and 2000 Census Adjustment Plans.” Technical Report 550, Department of Statistics, U.C. Berkeley.

Steffey, D.L. and N.M. Bradburn, editors (1994). Counting People in the Information Age. Washington, D.C.: National Academy Press.

Thompson, J. (1990). “Census Bureau Memorandum N-2 to Arnold Jackson.” U.S. Bureau of the Census, Washington, D.C.

U.S. Bureau of the Census (1990). "Technical Operational Plans for the 1990 PES." Washington, D.C.

U.S. Bureau of the Census (1991). "Census Bureau Releases Refined Estimates from Post- Enumeration Survey of 1990 Census Coverage.” Press Release (13 June 1991) CB91-221, Washington, D.C.

Wachter, K.W. (1992). "Variabilité aléatoire des phenomènes démographiques: enseignements des séries paroissiales de Wrigley et Schofield.” In A. Blum, N. Bonneuil, and D. Blanchet, editors. Modèles de la démographie historique. Paris: Institut National d'Etudes Démographiques, Presses Universitaires de France.

Wachter, K.W. and D.A. Freedman (2000). "The Fifth Cell: Correlation Bias in U.S. Census Adjustment." Evaluation Review, 24: 191-211.

Wolter, K. and B. Causey (1991). "Evaluation of Procedures for Improving Population Estimates for Small Areas." Journal of the American Statistical Association, 86: 278-284.

Zaslavsky, A.M. (1993). "Combining Census, Dual System, and Evaluation Study Data to Estimate Population Shares.” Journal of the American Statistical Association, 88: 1092-1105. 


\section{Appendix}

\section{A1 A Simple Binomial Model}

Formula $\{4\}$ is motivated by the following idea. Fix a territory and demographic group. Localities are indexed by $i=1, \ldots, L$. Focus on a particular property, e.g., living in multi-unit housing. Suppose people in that territory and group are independent, and in locality $i$ there is a common probability $p_{i}$ of having the property in question. Heterogeneity is amplified by binomial variation, and it is an estimate of binomial variation that is the correction term in $\{4\}$.

More particularly, from locality $i$ we choose a block at random and observe the $N_{i}$ persons in that block; $X_{i}$ persons have the property in question. Conditioned on the choice of blocks, the $X_{i}$ are independent binomial variables, with $N_{i}$ for the number of trials and success probability $p_{i}$. Now $\hat{p}_{i}=X_{i} / N_{i}$ and $\hat{p}=\sum_{i} \hat{p}_{i} / L$. Of course,

$$
\mathrm{E}\left(\hat{p}_{i}\right)=p_{i}, \quad \mathrm{E}(\hat{p})=\frac{1}{L} \sum_{i} p_{i}=p
$$

while

$$
\operatorname{var}\left(\hat{p}_{i}\right)=\frac{p_{i}\left(1-p_{i}\right)}{N_{i}}, \quad \operatorname{var}(\hat{p})=\frac{1}{L^{2}} \sum_{i} \frac{p_{i}\left(1-p_{i}\right)}{N_{i}}, \quad \operatorname{cov}\left(\hat{p}_{i}, \hat{p}\right)=\frac{1}{L} \frac{p_{i}\left(1-p_{i}\right)}{N_{i}}
$$

The expected value of the naive estimator $\{3\}$ is now easy to work out, and is

$$
\frac{1}{L} \sum_{i}\left(p_{i}-p\right)^{2}+\Delta, \quad \text { where } \quad \Delta=\frac{1}{L}\left(1-\frac{1}{L}\right) \sum_{i} \frac{p_{i}\left(1-p_{i}\right)}{N_{i}}
$$

is the excess binomial variance. Finally - by design - the expected value of the correction term in $\{4\}$ equals $\Delta$, just canceling the contribution from excess binomial variance.

\section{A2 Data-Dependent Areas}

Our measure $\hat{H}$ has simple properties in simple settings. If the local areas have fixed boundaries and samples of fixed numbers of individual post-stratum members are drawn from the local areas, then the theory just developed applies, and $\hat{H}^{2}$ is unbiased; the binomial formulas are easily adapted to simple random sampling. However, P-12 is not a simple setting. Data-dependent aggregation of blocks into superblocks, to be described shortly, implies local areas with random 
boundaries. The numbers of sampled individuals in these areas are themselves random, not fixed, and that leaves the correction term in the definition of $\hat{H}^{2}$ in need of justification. Sampling block clusters instead of individuals introduces a term for cluster-level heterogeneity into the expectations. We sketch our treatment of the data-dependence first and the term for clustered sampling next.

The data-dependent boundaries turn $p_{i}$ and $H$ into random quantities with expectations, and the goal is to justify the formulas

$$
\mathrm{E}\left(\hat{p}_{i}\right) \approx \mathrm{E}\left(p_{i}\right) \text { and } \mathrm{E}\left(\hat{H}^{2}\right) \approx \mathrm{E}\left(H^{2}\right)+\frac{1}{L} \sum_{i} d_{i} W_{i} .
$$

In the display, $W_{i}$ accounts for within-area between-cluster covariance and $d_{i}$ is the analog of a finite-sample correction factor. Both are defined below. We believe both are small, but our argument is only heuristic, and that is one reason why our conclusions in this paper are somewhat tentative.

The Census Bureau's aggregation process, merging sample blocks into sample superblocks, may be described as follows [Bateman 1991]. Within each post-stratum, after the P-12 sample has been drawn, members are pooled together from block after block, following the sequence of blocks in the sample list, until a minimum of ten members are included or a state boundary is reached. Post-strata represent a fine-grained subdivision of the population along demographic lines, so most blocks contain at most a handful of people from the same post-stratum. The stopping rule for superblock completion typically puts half a dozen blocks into a superblock.

The list for the sampling frame snakes its way through the territory spanned by the post-stratum from place to place among places of the same place type. The sampled blocks amalgamated into one sample superblock are therefore often but not always drawn from the same contiguous area. Superblocks are put together separately for each post-stratum and superblocks formed for different post-strata do not coincide.

For our formal arguments, we use the word "locality" for the local area defined to correspond to a particular superblock in the following way. Split the ordered list of blocks in the sampling frame randomly at a uniformly distributed point between the last sampled block in the previous superblock and the first sampled block in the current superblock. Repeat the procedure between the current superblock and the succeeding one. That gives two breakpoints. The locality corresponding to the current superblock is the set of all blocks in the list between the two breakpoints. The superblock then equals the subset of blocks in the locality selected into the sample.

The order in the sampling frame maintains the integrity of address-register areas and Census 
district office areas, so a locality is often a contiguous or nearly contiguous area, but not always so. The rate $p_{i}$ is calculated for all the members in all the blocks in the the sampling frame in the $i$ th locality. It is a random quantity because it depends on sample selection, on the operation of the stopping rule, and on the outcome of the splitting. The randomness in $p_{i}$ turns $H$ into a random quantity as well.

We can write $\hat{p}_{i}$ in the form

$$
\hat{p}_{i}=\frac{1}{N_{i}} \sum_{m} \xi_{m} J\left(c_{m} \in S\right) .
$$

In our notation,

$\xi_{m}$ is the binary outcome for the $m$ th member of the post-stratum in the $i$ th locality. For example, for multi-unit housing rates, $\xi_{m}$ equals 1 if the corresponding person lives in multi-unit housing and equals 0 else.

$c_{m}$ is the block cluster (P-12 sampling unit) to which the $m$ th member belongs.

$S$ is the set of clusters in the sample in the $i$ th superblock, $s$ in number; a subscript for $i$ is suppressed.

$J$ is the indicator function of a set.

The argument that $\mathrm{E}\left(\hat{p}_{i}\right) \approx \mathrm{E}\left(p_{i}\right)$ has four steps. First, we express $\mathrm{E}\left(\hat{p}_{i}\right)$ as the expectation of the conditional expectation given $N_{i}$, the number of members in the $i$ th superblock. Second, we argue that $\mathrm{E}\left(J\left(c_{m} \in S\right) \mid N_{i}\right)$ is nearly constant in $m$. That entails arguing against any sizable endpoint effects stemming from the random boundaries of the localities. It also entails arguing that conditioning on $N_{i}$ has little impact, inasmuch as the stopping rule produces values of $N_{i}$ that exceed the required minimum of 10 members per superblock only by the overshoot contributed by the last included block. Third, we count up terms with $\xi_{m}=0$ and $\xi_{m}=1$; the answers are familiar combinatorial expressions. Fourth, we argue that the people per cluster in the universe divided by the people per cluster in the sample should be close to unity and not strongly associated with $p_{i}$. That is enough to conclude that $\mathrm{E}\left(\hat{p}_{i}\right) \approx \mathrm{E}\left(p_{i}\right)$.

The same line of reasoning leads, with more effort, to an approximation for $\mathrm{E}\left[\left(\hat{p}_{i}-p_{i}\right)^{2}\right]$. Some terms coincide with the binomial-formula terms found in the definition of $\hat{H}$. One set of cross-product terms, involving clusters in different localities, cancels. Another set of cross-product terms, involving pairs of clusters in the same locality, contributes the terms $d_{i} W_{i}$ discussed in the next subsection.

These considerations are in principle further complicated by the fact that the PES and P-12 
samples are stratified samples with some variation in sampling weights. Sampling stratum membership is not indicated in the P-12 dataset. Sampling strata and sampling weights have major effects in the PES, but we expect their effects in P-12 to be minor for several reasons, including the absence of movers, the lack of non-response reweighting and special small-block samples, and the fact that our $\hat{p}$ and $p$ are not weighted averages but simple averages across localities.

\section{A3 Effects of Clustered Sampling}

The P-12 sample is a clustered sample primarily because individuals are clustered into blocks and secondarily because blocks are clustered into block clusters (containing one or two blocks in most cases). In the presence of clustered sampling, heterogeneity from cluster to cluster within localities makes a downweighted but nonzero contribution to sampling variability in $\sum\left(\hat{p}_{i}-\hat{p}\right)^{2}$ and introduces, as we have said, a term of the form $d_{i} W_{i}$ into $\mathrm{E}\left(\hat{H}^{2}\right)$. The average within-cluster covariance in the universe of members of the $i$ th locality is given by

$$
W_{i}=\left(\sum_{m \neq m^{\prime}}\left(X_{m}-p_{i}\right)\left(X_{m^{\prime}}-p_{i}\right) J\left(c_{m}=c_{m^{\prime}} \in U\right)\right) /\left(\sum_{c} M_{c}\left(M_{c}-1\right)\right)
$$

The sums range over all clusters in the $i$ th locality, and $M_{c}$ is the number of members in the $c$ th block cluster. The denominator is the number of terms in the numerator. For the contribution to sampling variability, $W_{i}$ must be multiplied by $d_{i}$, where

$$
d_{i}=\mathrm{E}\left(\sum_{c} M_{c}\left(M_{c}-1\right)\right) /\left(N_{i} \sum_{c} M_{c}\right)
$$

If members of the post-stratum were spread out with one member per cluster, $d_{i}$ would be zero. If each cluster always had 10 members, forcing $M_{c}=N_{i}=10$ under the stopping rule and creating single-cluster superblocks, $d_{i}$ would be $9 / 10$. (With our notation, if the $i$ th superblock in the sample has index $c$ in the sampling frame, then $N_{i}=M_{c}$.)

The covariance factor $W_{i}$ measures how much more often the outcomes for two members of the same cluster agree compared to the outcomes for two randomly chosen members of the whole locality. At the extreme, each cluster could consist entirely of ones or entirely of zeros, irrespective of size, and then we would have $W_{i}=p_{i}\left(1-p_{i}\right)$, the variance of the outcome for a single randomly-selected member of the locality. The downweighting $d_{i}$ would scale this variance by a kind of effective sample size for the clustered sampling. Usually, however, knowing $X_{m}$ gives only limited information about $X_{m^{\prime}}$, and $W_{i}$ will be close to zero. 
The only non-zero contributions to $W_{i}$ come from clusters with two or more members; large contributions only from clusters with many members. Clusters with many members appear to be rare. The identity of blocks is erased in the P-12 data set; however, we have detailed census and PES data for metropolitan areas outside central cities in the Pacific division, nicknamed the "Berkeley data set." In these data, of the clusters that contain any post-stratum member, about $20 \%$ contain only one such person. (We are averaging over post-strata.) Another $16 \%$ contain 2 people, and only about $20 \%$ contain 7 or more. The $d_{i}$ factors average out near $1 / 2$.

We cannot measure $W_{i}$ directly from P-12, and the PES sample is much too small for stable estimates. There is, however, an empirical test of the extreme hypothesis that all or most of the observed values of $\hat{H}^{2}$ are contributed by within-cluster covariances. Under this hypothesis, $W_{i}$ would not increase as localities and superblocks are merged into superlocalities and supersuperblocks, and $d_{i}$ would decrease in accordance with the formula $\{11\}$. Values of $\hat{H}$ have been inspected under a sequence of mergings for selected post-strata: $\hat{H}$ falls off substantially more slowly than its predicted value under this extreme hypothesis. Any other outcome would be surprising; the small numbers of post-stratum members per cluster makes the sampling quite close to random sampling of individuals and thus to the case where the within-cluster covariance contribution is absent.

Both between-locality heterogeneity and between-cluster within-locality heterogeneity are forms of heterogeneity. $\hat{H}$ remains a measure of heterogeneity whether or not the $W_{i}$ contributions are small. But between-locality heterogeneity is of primary interest; it is the contribution which directly affects estimates for whole local areas. The arguments in this section support the view that in the P-12 data set the approximation $\mathrm{E}\left(\hat{H}^{2}\right) \approx \mathrm{E}\left(H^{2}\right)$ is a workable one, and that the values in Table 1 are principally to be interpreted as evidence of heterogeneity from locality to locality.

\section{A4 The Standard Errors for Table 1}

The nine Census divisions represent, with a handful of exceptions, disjoint groups in the sampling scheme and their RMS $\hat{H}^{2}$ s are essentially independent of each other. The squared measure $\hat{H}^{2}$ in Table 1 is the weighted mean of the nine $\hat{H}^{2}$ s for the divisions, weighting by the number of poststrata in each division. For our calculation we make the assumption that the expected values of the nine measures for the divisions are all the same (cf. Table 4), while the nine variances differ. We write down an unbiased estimator for the variance of the overall measure as a weighted average of the squared deviations of the divisional measures from the overall weighted mean. The weights are functions of the numbers of post-strata in the divisions. This estimate should be something of an 
upper bound, because part of the variability in divisional measures must reflect small differences among expected values rather than sampling variability as assumed. We convert to square roots with a delta-method approximation.

Two alternative estimates for the sampling standard errors in $\hat{p}$ are given in Table 1 . An indirect approach is required because the identity of the sampling units has been erased by the superblock aggregation process; to our knowledge, the Census Bureau has not published direct estimates of standard errors for P-12. The low estimate in Table 1 is obtained by treating individuals as if they were the sampling units; the sampling variance for a post-stratum-wide $\hat{p}$ is then computed as $\hat{p}(1-\hat{p}) /\left(\sum N_{i}\right)$. The high estimate treats superblocks as if they were the sampling units. Then the sampling variance is computed as

$$
\frac{1}{L(L-1)} \sum_{i}\left(\hat{p}_{i}-\hat{p}\right)^{2}
$$

where $L$ is the number of super-blocks associated with the post-stratum in question. These estimates apply to P-12 itself; finally, we rescale in proportion to sample size, as measured by block clusters. 